\title{
Infección Latente por Tuberculosis en Trabajadores de Salud en Guayaquil, Ecuador.
}

\section{Latent Tuberculosis Infection in Health Workers in Guaya- quil, Ecuador}

Sunny Sánchez-Giler ${ }^{a}$, Dolores Zambrano-Castro ${ }^{b}$, Debbie Nath-Lynch ${ }^{c}$, Andrea Murillo-Soriano ${ }^{\text {, Juan }}$ Murillo-Zambrano ${ }^{e}$, Gregorio Ortiz-Bermudez ${ }^{f}$, Ma. Fernanda Sánchez-Carriel ${ }^{g}$, Roberto Oleas-Narea ${ }^{h}$, Genaro Cedeño-Gutiérrezi, Walter Noboa-Quimí 'j, Doménica Sotomayor-Salvatierrak, Doménica

Santamaría-Obando

INFORMACIÓN DEL ARTÍCULO

Fecha de recepción:10 de febrero del 2017

Fecha de aceptación: 22 de marzo del 2017

${ }^{a}$ Magíster en Microbiología. Docente Universidad Espíritu Santo - Ecuador E-mail:sunsanchez@uees.edu.ec

$b$ Universidad Espíritu Santo Ecuador.

c Universidad Espíritu Santo Ecuador.

$d$ Universidad Espíritu Santo Ecuador.

e Universidad Espíritu Santo Ecuador.

$f$ Universidad Espíritu Santo Ecuador.

$g$ Universidad Espíritu Santo Ecuador.
Resumen

Infección latente por tuberculosis y tuberculosis activa representan un riesgo ocupacional para los trabajadores de salud, quienes están expuestos a pacientes infectados en sus lugares de trabajo. Este riesgo es particularmente serio para los trabajadores de salud en Guayaquil, Ecuador debido a la alta incidencia de tuberculosis en esta ciudad. El presente artículo busca determinar la prevalencia de LTBI en trabajadores de salud en Guayaquil, Ecuador; a través de un estudio transversal y no experimental conducido durante Junio, 2013-Mayo, 2014 en 124 trabajadores de salud de unidades hospitalarias públicos y privados. Se completó una encuesta epidemiológica y se obtuvo muestras de sangre. Se ejecutó el ensayo Quantiferon Tb Gold in-Tube. La ausencia de síntomas de TB activa confirmó el diagnostico de LTBI. Entre los hallazgos: 37 de los 124 participantes, o $29,80 \%$, fueron positivos para LTBI por el test de IGRA. La mayoría de los individuos positivos se ubicaron entre los 41-50 años de edad (10,5\%) y fueron del género femenino $(21,0 \%)$. La prevalencia de LBTI en trabajadores de salud en Guayaquil fue $29.80 \%$, similar a los valores reportados en otros estudios de ciudades que comparten características similares.

\section{Palabras Clave}

Tuberculosis activa, LTBI, IGRA, trabajadores de salud, riesgo ocupacional.

Clasificación JEL: I10, I18.

Contribuciones de autor

SSG ha participado en la conceptualización del estudio, formulación del método para análisis estadístico y análisis utilizando el software Epi Info 7. DZC ha participado en la formulación de métodos para análisis estadístico, análisis utilizando el software Epi Info 7, redacción de documentos. DNL, AMS, JMZ, GOB, MSC, RON, GCG, WNQ, DSS, DSO, DZC participaron en la toma de muestra de sangre, realización pruebas IGRA y encuesta epidemiológica a los participantes. DNL, AMS, MSC traducción de manuscrito. Todos los autores han leído y aprobado el manuscrito final.

\section{Financiamiento}

Los autores agradecen el apoyo financiero de la Universidad Espíritu Santo.

\section{Conflicto de Interés}

Todos los autores declaran no poseer conflictos de interés relevantes para este artículo. 


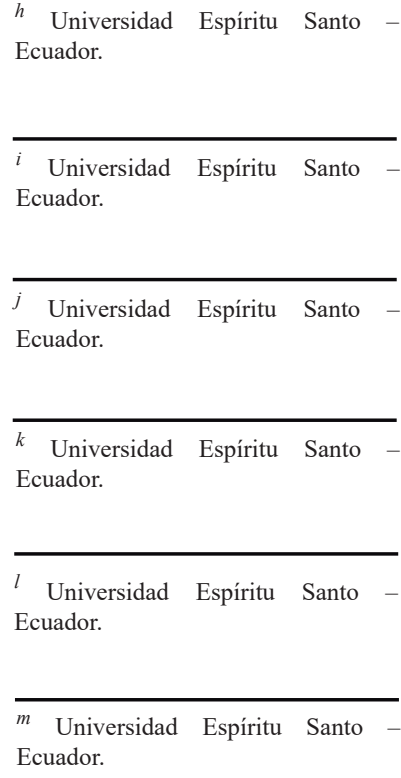

\begin{abstract}
Latent tuberculosis infection (LTBI) and active tuberculosis represent an occupational risk for health workers, who are exposed to infected patients in their workplaces. This risk is particularly serious for health workers in Guayaquil, Ecuador because of the high incidence of tuberculosis in this city. The objective of the paper is to determine the prevalence of LTBI in health workers in Guayaquil, Ecuador; this determination was conducted through a cross-sectional and non-experimental study done from June, 2013 to May, 2014 in 124 health workers from public and private hospitals. An epidemiological survey was completed and blood samples were collected. The Quantiferon Tb Gold in-Tube essay was run. The absence of symptoms of active TB confirmed the diagnosis of LTBI. Among the findings: 37 of the 124 participants, or $29.80 \%$, were positive for LTBI by the IGRA test. Most of the positive individuals were between $41-50$ years of age (10.5\%) and 21.0\% were female (The prevalence of LBTI in health workers in Guayaquil was $29.80 \%$, similar to the values reported in other studies in cities that share similar characteristics.

\section{Keywords}

Active tuberculosis, LTBI, IGRA, healthcare workers, occupational risk.
\end{abstract}

JEL Classification: I10, I18.

\section{Introducción}

La tuberculosis activa (TB) en la era de VIH/sida, es la segunda causa de muerte más frecuente debida a agentes infecciosos; con una habilidad considerable para establecer una infección latente, que disminuye el control de la propagación de la infección (Dagnew et al., 2012). Se estima que a nivel mundial, 5.4 millones de personas adquirieron TB en el 2014 (OMS, 2014). La exposición a una persona con TB activa conlleva a infección que puede evolucionar a la eliminación del bacilo tuberculoso por el sistema inmune innato, la producción de infección primaria al escapar de este sistema o al desarrollo de infección latente por tuberculosis (LTBI, por sus siglas en inglés) (Ahmad, 2011).
LTBI es el resultado de una respuesta inmunitaria persistente a los antígenos de Mycobacterium tuberculosis, sin la presencia de manifestaciones clínicas (Sharma, Mohanan, \& Sharma, 2012). Aproximadamente un tercio de la población mundial ostenta LTBI, con tasas de prevalencia de hasta $79 \%$, exhibiendo un riesgo anual de desarrollo de TB activa del $0.5 \%$; valor que puede llegar hasta el $14.3 \%$ en los trabajadores de salud (TS), debido a su exposición constante y por mayor tiempo al microorganismo (Rafiza, Rampal, \& Tahir, 2011; Sharma et al., 2012). Es así que la LTBI y TB representan un riesgo ocupacional en TS. El nivel de educación, compartir vivienda con individuos infectados con TB, estado nutricional, abusos de sustancias, entre otros, representan otros factores de riesgo (Shakak et al., 2013). 
La Organización Mundial de la Salud (OMS) ha ubicado al Ecuador en tercer lugar de mayor frecuencia de infección entre los países de la Región, con una incidencia de TB de 59, prevalencia de 98 y tasa de mortalidad de 2.7 por cada 100.000 habitantes (OMS, 2014). Además, en el año 2014 la Organización Panamericana de la Salud (OPS) reportó que la provincia del Guayas, específicamente la ciudad de Guayaquil, poseía el más alto número de casos de TB $(70 \%$ del total se encontraban en este lugar) (OPS, 2014).

Previamente, el diagnóstico de LTBI se realizaba únicamente con la prueba de tuberculina, que mide la respuesta de hipersensibilidad hacia el derivado proteico purificado (PPD, por sus siglas en inglés), que contiene una mezcla de antígenos del complejo M. tuberculosis, $M$. bovis y algunas otras micobacterias no tuberculosas como $M$. leprae, $M$. africanun, $M$. avium, entre otras. Posee una sensibilidad estimada de $100 \%$ en individuos con LTBI. No obstante, presenta una alta tasa de falsos positivos, por ejemplo: en personas infectadas con micobacterias no tuberculosas (muchas de ellas forman parte de la PPD); inmunización previa con vacuna de Bacillus Calmette-Guerin (BCG), que consiste en una preparación de bacterias vivas atenuadas a partir de Mycobacterium bovis y forma parte del programa nacional de vacunación obligatorio, cuya aplicación está indicada durante las primeras 24 horas de vida; o, debido a un efecto infligido por pruebas repetitivas. Todo esto se traduce en una baja especificidad (88\%), con un valor predictivo positivo y valor predictivo negativo del $95 \%$ y $86 \%$, respectivamente (Lee, Kim, \& Lee, 2011; Menzies, 2000; Regatieri, Abdelwahed, Perez, \& Bush, 2011).

Se han desarrollado técnicas diagnósticas para LTBI, como ensayos de liberación de interferón gamma (IGRA) (Ringshausen, Schablon, \& Nienhaus, 2012). El más conocido es Quantiferon Tb Gold in-Tube (QTF-GIT), una técnica de ELISA empleada para medir la producción de interferón gamma (IFN- $\gamma$ ) por células $\mathrm{T}$ circulantes frente a antígenos ESAT-6 (early secreted antigen target 6), CFP-10 (cultura filtrade protein 10) y una porción del antígeno TB7.7 de M. tuberculosis, los cuales se encuentran ausentes en las cepas de BCG y otras micobacterias no tuberculosas. Exhibe una especificidad de 95\% y sensibilidad de $80 \%$ (Pai, Zwerling, \& Menzies, 2008). Sin embargo, se recomienda esta técnica en países donde los individuos reciben la BCG (CDC, 2013).

El presente estudio buscó establecer la prevalencia de LTBI en trabajadores de salud en centros de salud público y privados de la Ciudad de Guayaquil, pues representan grupos de alto riesgo para esta infección.

\section{Métodos}

\section{Consideraciones Científicas y Éticas}

El estudio fue aprobado y revisado 
Sunny Sánchez-Giler, Dolores Zambrano-Castro, Magister en Microbiología. Docente Universidad, Debbie Nath- Lynch , Andrea Murillo-Soriano, Juan Murillo-Zambrano, Gregorio Ortiz-Bermudez, Ma. Fernanda Sánchez-Carriel, Roberto Oleas-Narea, Genaro Cedeño-Gutiérrez, Walter Noboa-Quimí, Doménica Sotomayor-Salvatierra, Doménica Santamaría-Obando

por el Comité de Ética de la Clínica Kennedy, que se encuentra aprobado por la Autoridad Sanitaria Nacional. Se obtuvo el consentimiento informado escrito de todos los individuos participantes, además se utilizaron códigos numéricos para mantener la confidencialidad de los resultados.

\section{Diseño de estudio y muestra}

Estudio transversal, no experimental, de prevalencia, conducido entre Junio del 2013 a Mayo del 2014, en trabajadores de la Salud, quienes laboran en 2 hospitales públi$\cos$ y 1 subcentro de salud de la ciudad Guayaquil, ubicados en zonas del centro y sur.
Los participantes del estudio incluyeron: enfermeras, asistentes médicos, técnicos de laboratorio médico, personal de servicio, médicos tratantes y personal de oficina acorde a la categorización del Anuario de Recursos y Actividades de Salud (Yunga, 2014). Los criterios de inclusión establecidos fueron: firma de consentimiento informado, mayoría de edad, muestra suficiente para prueba ELISA y datos completos en la ficha; se excluyeron individuos con síntomas de TB activa como: dificultad respiratoria, tos, expectoración con sangre, sudoración excesiva sobre todo en la noche, fatiga, fiebre, pérdida de peso (sintomáticos respiratorio) (Cecil \& Goldman, 2013). El cálculo de la muestra se realizó de la siguiente forma:

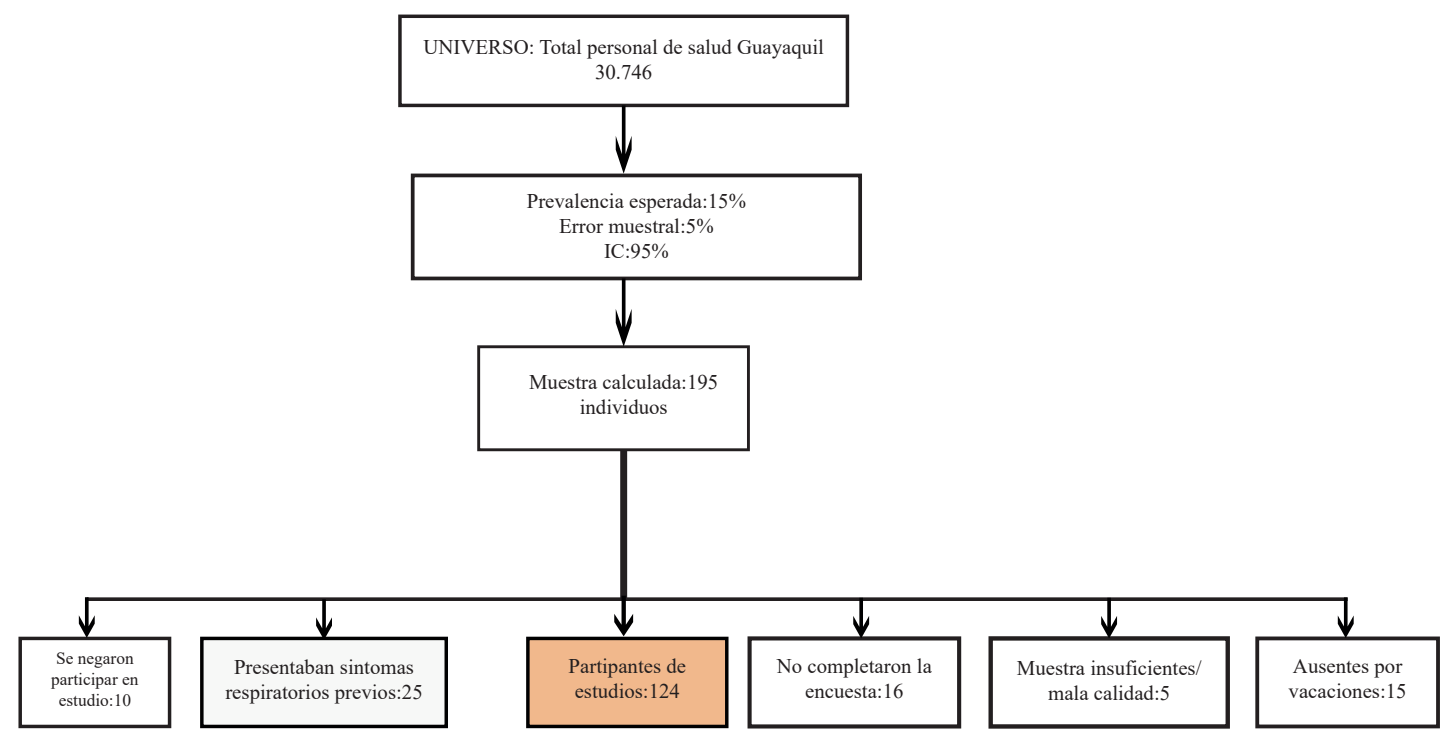

Figura 1. Proceso de selección de participantes del estudio. El universo fue tomado de: Yunga J. Anuario de Estadística de Salud: Recurso y Actividades 2014. Instituto Nacional de Estadísticas y Censos. 2014 


\section{Recolección de Datos}

Se obtuvo información correspon- diente a factores de riesgo: socio- demográficos, historia laboral, comorbilidades, sustancias de abuso, historia previa de TB e historia de convivencia en el mismo espacio con familiares o amigos diagnosticados con TB. Además, se recolectó 5 $\mathrm{ml}$ de sangre en 3 tubos que contienen antígenos representativos de proteínas de M. tuberculosis y controles: antígeno TB, control nulo y control mitógeno.

\section{Ejecución del Ensayo}

Las muestras obtenidas fueron incubadas a $37^{\circ} \mathrm{C}$ por un período de 16 a 24 horas, con una centrifugación posterior; luego de ello, se ejecutó la prueba acorde a las instrucciones del fabricante.

Los resultados fueron interpretados por el software de análisis de QFT TB Git. La prueba es considerada positiva cuando el antígeno TB estimula niveles plasmáti$\cos$ de IFN- $\gamma \geq 0.35 \mathrm{IU} / \mathrm{mL}$. Una respuesta inferior a $0.5 \mathrm{IU} / \mathrm{ml}$ por parte del mitógeno se considera un resultado indeterminado.

\section{Análisis de Datos}

La información obtenida se almacenó en una base de datos de Excel y se analizó mediante el software estadístico SPSS Statistics 22. Se aplicó medidas de frecuencia y asociación de los diversos factores de riesgo.

\section{Resultados}

Participaron 124 individuos en la investi- gación, 82 corresponden al género femenino $(66,1 \%)$ y 42 al género masculino (33,9\%). Siendo de 21-30 años el grupo etáreo más frecuente $(39,5 \%)$, seguido por 31-40 años (25,0\%). Según su ocupación se dividió de la siguiente forma: 60 médicos, 17 enfermeras, 15 técnicos de laboratorio, 11 asistentes de laboratorio, 11 personal administrativo y 10 personal de servicio.

La prevalencia de LTBI fue de $29,80 \%$. Se evidencia que el grupo etáreo con mayor frecuencia de positividad para IGRA corresponde a 41-50 años (10,5\%), seguido de 31-40 años $(9,7 \%)$. En relación al género, 26 corresponden al sexo femenino $(21,0 \%)$ y 11 al sexo masculino $(8,9 \%)$. Se encuentran casados 15 de ellos $(12,1 \%)$ y 15 solteros $(12,1 \%), 23$ han cursado estudios universitarios $(18,5 \%)$, mientras $26,6 \%$ reportan trabajar un promedio de 8 horas al día.

La mayoría de los participantes positivos no vivían en condiciones que pudieran aumentar el riesgo de TB o LTBI. 30 de ellos viven en viviendas propias, cuyos materiales de construcción varían entre zinc para el techo y cemento para las paredes. Por otro lado, $17,7 \%$ indicaron vivir con un promedio de 4-6 personas en sus casas y dormir solos en sus habitaciones. Además el $100 \%$ de los participantes refieren contar con los servicios básicos, que constan de agua potable, energía eléctrica y alcantarillado. 6 refirieron tener familiares con diagnóstico de TB y 15 reportaron tener compañeros con infección por TB. 
Los individuos con LTBI reportaron comorbilidades que incluyen hipertensión, diabetes mellitus, asma; aunque en su mayoría indicó que no posee patologías asociadas (16,1\%). Además el $11,11 \%$ refirieron que fumaban hace 3 a 4 años, un promedio de 5 cigarrillos por día. En contraste el 30,56\% reporta que consumen alcohol con una cronicidad de 20 años de consumo ocasional. De igual forma, el análisis de asociación con la Prueba Chi cuadrado de Pearson evidenció una relación estadísticamente significativa entre las variables edad, nivel de educación y reporte de tener compañeros de trabajo con $\mathrm{TB}$, y la infección. El análisis estadístico se detalla en la tabla 1.

Tabla 1.

Características socio-demográficas y otros factores de riesgo en participantes de estudio

\begin{tabular}{lccccccc} 
& \multicolumn{3}{c}{ IGRA +} & \multicolumn{2}{c}{ IGRA - } & \multicolumn{2}{c}{ Chi-Cuadrado de Pearson } \\
\hline VARIABLES & $\#$ & $\%$ & $\#$ & $\%$ & Valor & gl & Sig. asintótica (2 caras) \\
\hline Sexo & & & & & & & \\
\hline Masculino & 11 & 8,9 & 31 & 25,0 & & &, 525 \\
Femenino & 26 & 21,0 & 56 & 45,2 &, $404^{\mathrm{a}}$ & 1 & \\
TOTAL & 37 & 29,9 & 87 & 70,2 & & & \\
\hline Edad & & & & & & & \\
\hline 18-20 años & 2 & 1,6 & 6 & 4,8 & & & \\
21-30 años & 6 & 4,8 & 43 & 34,7 & & & \\
31-40 años & 12 & 9,7 & 19 & 15,3 & & & \\
41-50 años & 13 & 10,5 & 10 & 8,1 & $16,658^{\mathrm{a}}$ & 5 & \\
51-60 años & 2 & 1,6 & 6 & 4,8 & & & \\
61-70 años & 2 & 1,6 & 3 & 2,4 & & & \\
TOTAL & 37 & 29,8 & 87 & 70,1 & & & \\
\hline
\end{tabular}

\section{Estado civil}

\begin{tabular}{lccccccc}
\hline Casado & 15 & 12,1 & 28 & 22,6 & & & \\
Unión libre & 3 & 2,4 & 7 & 5,6 & & &, 609 \\
Viudo & 1 & 0,8 & 1 & 0,8 & $2,703^{\text {a }}$ & 4 & \\
Soltero & 15 & 12,1 & 35 & 28,2 & & & \\
Divorciado & 3 & 2,4 & 16 & 12,9 & & & \\
TOTAL & 37 & 29,8 & 87 & 70,1 & & &
\end{tabular}




\begin{tabular}{|c|c|c|c|c|c|c|c|}
\hline \multicolumn{8}{|l|}{ Zona que habita } \\
\hline Urbana & 37 & 29,8 & 86 & 69,4 & \multirow{3}{*}{, $429^{a}$} & \multirow{3}{*}{1} & \multirow{3}{*}{, 513} \\
\hline Rural & 0 & 0,0 & 1 & 0,8 & & & \\
\hline TOTAL & 37 & 29,8 & 87 & 70,2 & & & \\
\hline \multicolumn{8}{|c|}{ Nivel de educación } \\
\hline Analfabeto & 0 & 0,0 & 1 & 0,8 & \multirow{7}{*}{$11,077^{\mathrm{a}}$} & \multirow{7}{*}{5} & \multirow{7}{*}{, 050} \\
\hline Primaria & 1 & 0,8 & 9 & 7,3 & & & \\
\hline Secundaria & 4 & 3,2 & 18 & 14,5 & & & \\
\hline Técnico medio & 1 & 0,8 & 0 & 0,0 & & & \\
\hline Técnico superior & 8 & 6,5 & 6 & 4,8 & & & \\
\hline Universidad & 23 & 18,5 & 53 & 42,7 & & & \\
\hline TOTAL & 37 & 29,8 & 87 & 70,1 & & & \\
\hline \multicolumn{8}{|l|}{ Propiedad (Casa) } \\
\hline Propia & 30 & 24,2 & 65 & 52,4 & \multirow{4}{*}{, $842^{\mathrm{a}}$} & \multirow{4}{*}{2} & \multirow{4}{*}{,656 } \\
\hline Alquilada & 3 & 2,4 & 12 & 9,7 & & & \\
\hline Prestada & 4 & 3,2 & 10 & 8,1 & & & \\
\hline TOTAL & 37 & 29,8 & 87 & 70,2 & & & \\
\hline
\end{tabular}

Número de personas que viven en la casa

\begin{tabular}{lccccccc}
\hline $1-3$ personas & 12 & 9,7 & 19 & 15,3 & & & \\
4-6 personas & 22 & 17,7 & 55 & 44,4 & & &, 339 \\
Más de 6 & & & & & $2,164^{\mathrm{a}}$ & 2 & \\
personas & 3 & 2,4 & 13 & 10,5 & & & \\
TOTAL & 37 & 29,8 & 87 & 70,2 & & &
\end{tabular}

Número de personas que comparten habitación

\begin{tabular}{lccccccc}
\hline 1 personas & 22 & 17,7 & 42 & 33,9 & & & \\
2 personas & 13 & 10,5 & 41 & 33,1 & & & \\
3 personas & 1 & 0,8 & 1 & 0,8 & $3,113^{\mathrm{a}}$ & 4 &, 539 \\
4 personas & 1 & 0,8 & 1 & 0,8 & & &
\end{tabular}


Sunny Sánchez-Giler, Dolores Zambrano-Castro, Magister en Microbiología. Docente Universidad, Debbie Nath- Lynch , Andrea Murillo-Soriano, Juan Murillo-Zambrano, Gregorio Ortiz-Bermudez, Ma. Fernanda Sánchez-Carriel, Roberto Oleas-Narea, Genaro Cedeño-Gutiérrez, Walter Noboa-Quimí, Doménica Sotomayor-Salvatierra, Doménica Santamaría-Obando

$\begin{array}{lcccc}5 \text { personas } & 0 & 0,0 & 2 & 1,6 \\ \text { TOTAL } & 37 & 29,8 & 87 & 70,2\end{array}$

\section{Relacionado con conocidos con TB}

\begin{tabular}{lccccccc}
\hline SI & 6 & 4,8 & 13 & 10,5 & & & \\
NO & 31 & 25,0 & 74 & 59,7 &, $032^{\mathrm{a}}$ & 1 &, 857 \\
TOTAL & 37 & 29,8 & 87 & 70,2 & & & \\
\hline
\end{tabular}

\section{Compañero de trabajo con TB}

\begin{tabular}{llllllll}
\hline SI & 15 & 12,1 & 10 & 8,1 & & & \\
NO & 22 & 17,7 & 77 & 62,1 & $13,607^{\mathrm{a}}$ & 1 &, 000 \\
TOTAL & 37 & 29,8 & 87 & 70,2 & & &
\end{tabular}

\section{Horas que trabaja al día}

\begin{tabular}{lccccccc}
\hline 4 a 8 horas & 33 & 26,6 & 78 & 62,9 & & & \\
9 a 12 horas & 4 & 3,2 & 8 & 6,5 & & &, $496^{\mathrm{a}}$ \\
Más de 12 horas & 0 & 0,0 & 1 & 0,8 & & &, 780 \\
TOTAL & 37 & 29,8 & 87 & 70,2 & & & \\
\hline
\end{tabular}

\section{Tabaco}

\begin{tabular}{lccccccc}
\hline SI & 4 & 3,2 & 13 & 10,5 &, $375^{\text {a }}$ & 1 &, 541 \\
\hline TOTAL & 37 & 29,8 & 87 & 70,2 & & & \\
\hline
\end{tabular}

\begin{tabular}{lccccccc}
\hline Alcohol & & & & & & & \\
\hline SI & 12 & 9,7 & 34 & 27,4 & & &, 483 \\
NO & 25 & 20,2 & 53 & 42,7 &, $492^{\mathrm{a}}$ & 1 & \\
TOTAL & 37 & 29,9 & 87 & 70,1 & & & \\
\hline
\end{tabular}

\section{Discusión}

En este estudio se evalúa el ensayo QTF-GIT para diagnóstico de LTBI en una ciudad con altas tasas de tuberculosis y que considera la vacunación con BCG desde el nacimiento. Se ha constatado la utilidad de esta técnica en poblaciones con las características mencionadas ( Pai, Dendukuri, et al., 2008) y las observaciones obtenidas coinciden con lo reportado en otras investigaciones con similar metodología (Nienhaus, Schablon, Preisser, Ringshausen, \& Diel, 2014). 
La prevalencia encontrada fue $29,80 \%$, valor que se asemeja a lo reportado en investigaciones de diversos países en similares condiciones. Joshi et al. realizó una revisión sistemática en la que se reportó una prevalencia de LTBI entre $33 \%$ a $79 \%$ en países con ingresos de bajos a medios (Joshi, Reingold, Menzies, \& Pai, 2006); así mismo, Whitaker et al. reportó una prevalencia de $31 \%$ en Georgia, en TS no expuestos a pacientes con TB prioritariamente, mientras que en aquellos expuestos a pacientes con TB activa fue del 55\% (Whitaker et al., 2013). Legesse et al. estimó una prevalencia de 63.7\% usando QTF-GIT (Legesse et al., 2011). No obstante, estos estudios también emplearon la prueba PPD, corroborándose una menor detección de LTBI con ella, aunque se reporta una buena concordancia entre QTF-GIT y la PPD. Por otro lado, la variabilidad en la prevalencia reportada, se atribuye a las diferencias entre la población de estudio de los diversos países, áreas geográficas y factores de riesgos a lo que se encuentran expuestos.

Repetidamente, se ha descrito que la población masculina presenta mayor frecuencia de LTBI (Shakak et al., 2013; Winje et al., 2008). No obstante, dicha situación no se cumple en este estudio donde la mayor prevalencia de LTBI se dio en individuos de sexo femenino. Similar a lo que reporta un estudio transversal realizado en tres grandes hospitales de la región occidental de Noruega, con mayor prevalencia de LTBI en participantes de sexo femenino (Gran, Assmus, \& Dyrhol-Riise, 2013). Sin embargo, la alta frecuencia en el sexo femenino en el presente estudio podría deberse a que gran parte de los participantes fueron de sexo femenino. No obstante, se ha indicado en diversos estudios una similitud en la proporción $\mathrm{H}: \mathrm{M}$, predominando los participantes del género femenino.

Al describir las características sociodemográficas de los individuos positivos se evidencia que mayormente se ubica entre los 31-50 años, con igual proporción entre casados y solteros, con un nivel de educación superior, lo que demuestra que este grupo está mayormente expuesto; además no manifiestan evidencia de hacinamiento y habitan en zonas urbanas.

Un factor de riesgo relevante es compartir espacios estrechos con personas que presenten la infección; sean familiares, amigos o pacientes. Franchi et al. evaluó riesgo de brotes de tuberculosis en salas obstétricas en Italia, donde determinó un mayor riesgo de brote por exposición sin protección en espacios cerrados sin ventilación (Franchi, Richeldi, Parrinello, \& Franco, 2007). Además Rafiza et al, también lo estableció como un factor fuertemente asociado al desarrollo de LTBI según el análisis de los resultados de su estudio (Rafiza et al., 2011). Estos resultados han sido similares en los establecidos por otros estudios (Haley et al., 2008; Hauck, Neese, Panchal, \& El-Amin, 2009). Sin embargo, según los datos de las encuestas gran parte de los participantes a pesar de convivir con individuos con infección activa, tenían espacios amplios y suficientes vías de ventilación como: ventanas, habitaciones para una sola persona, materiales adecua- 
Sunny Sánchez-Giler, Dolores Zambrano-Castro, Magister en Microbiología. Docente Universidad, Debbie Nath- Lynch , Andrea Murillo-Soriano, Juan Murillo-Zambrano, Gregorio Ortiz-Bermudez, Ma. Fernanda Sánchez-Carriel, Roberto Oleas-Narea, Genaro Cedeño-Gutiérrez, Walter Noboa-Quimí, Doménica Sotomayor-Salvatierra, Doménica Santamaría-Obando

dos de construcción, etc. Lo que indica que este no fue un factor de riesgo predominante para los participantes de este estudio.

De igual forma, se ha considerado una relación entre la prevalencia de LTBI y el período de trabajo (Joshi et al., 2006). Pai et al. realizó un estudio donde mostró una correlación entre la prevalencia de LTBI con una mayor duración del empleo, puesto que determinó que aquellos trabajadores de más de 10 años tenían un aumento de tres veces a más la prevalencia de LTBI comparado con aquellos que tenían menos de 6 años, asociando estos resultados con jornadas laborales prolongadas que se traduce en un mayor tiempo de exposición a pacientes con TB activa, aumentado la probabilidad de LTBI (Pai et al., 2005). Además Schablon el al. reportó un mayor riesgo de LTBI en los TS con mayor tiempo en sus puestos de trabajos, relacionado con mayor exposición a TB en un estudio transversal en un hospital para enfermedades pulmonares en Alemania (Schablon, Beckmann, Harling, Diel, \& Nienhaus, 2009). En esta investigación no se evidenció una asociación significante entre el tiempo de labor y LTBI, esto quizá porque el riesgo de infección tiene mayor relación con la concentración de partículas de Mycobacterium tuberculosis en el aire y la duración de la exposición de la persona (Rafiza et al., 2011).

Por otro lado se ha documentado que el abuso de sustancias sean estas alcohol, tabaco o drogas pueden contribuir a la infección por TB, ya que se ha establecido compromiso del estado general del paciente por su consumo excesivo. Estas sustancias pueden alterar el estado inmunológico y nutricional, perjudicando el estado general del individuo; lo que permite mayor predisposición a infecciones (Nienhaus et al., 2014). Según el análisis de la encuesta no se evidenció gran abuso de sustancias entre los participantes positivos; puesto que indicaron un consumo moderado de tabaco y alcohol, con poco tiempo de cronicidad, mientras no revelaron consumo de drogas.

Además, en últimas investigaciones se ha revelado que el tabaco tiene efectos negativos sobre la prueba IGRA, puesto que puede disminuir la liberación de IFN- $\gamma$ generando falsos negativos (van Zyl Smit et al., 2010). No obstante, esa posibilidad se reduce en este estudio, ya que su consumo es mínimo según los reportes de las encuestas epidemiológicas.

\section{Conclusiones}

Guayaquil es una ciudad catalogada por altos índices de infecciones por Mycobacterium tuberculosis, por lo que se han generado planes de prevención, control y tratamiento para individuos que presenten la patología. Situación que expone a TS que laboran dichas áreas, aumentando el riesgo de LTBI en este grupo poblacional.

La prevalencia de LTBI en trabajadores de la Salud que laboran en diferentes centros de salud de la Cuidad de Guayaquil fue de $29,80 \%$. La frecuencia de edad más afectada fue de 41 a 50 años y en el género femenino se encontró una mayor 
prevalencia. Para los individuos positivos para Quantiferon Tb Gold existe igual proporción entre casados y solteros, tienen estudios universitarios y poseen buenas condiciones habitacionales y de servicios básicos. Se recomienda desarrollar estudios a mayor escala y correlacionar los resultados de la técnica con datos clínicos e imagenológicos.

\section{Referencias}

Ahmad, S. (2011). Pathogenesis, Immunology, and Diagnosis of Latent Mycobacterium tuberculosis Infection. Clinical and Developmental Immunology.

CDC. (2013). Pruebas de detección de tuberculosis. Recuperado de https://www.cdc.gov/TB/esp/pd$\mathrm{f} / \mathrm{P}$ r u e b a s - d e - d e t e c ci\%C3\%B3n-de-tuberculosis.pdf

Cecil, \& Goldman, L. (2013). Tratado de Medicina Interna (24a ed., Vol. 1). España: Elsevier.

Dagnew, A. F., Hussein, J., Abebe, M., Zewdie, M., Mihret, A., Bedru, A., \& Aseffa, A. (2012). Diagnosis of latent tuberculosis infection in healthy young adults in a country with high tuberculosis burden and $\mathrm{BCG}$ vaccination at birth. BMC Research Notes, 5, 415. http://doi.org/10.1186/17 $56-$ 0500-5-415

Franchi, A., Richeldi, L., Parrinello, G., \& Franco, G. (2007). Room size is the major determinant for tuberculin conversion in health care workers exposed to a multidrug-resistant tuberculosis patient. International Archives of Occupational and Environmental Health, 80(6), 533-538. https://doi.org/10.1007/s00420-006-0160-1

Gran, G., Assmus, J., \& Dyrhol-Riise, A. M. (2013). Screening for latent tuberculosis in Norwegian health care workers: high frequency of discordant tuberculin skin test positive and interferon-gamma release assay negative results. BMC Public Health, 13, 353. h t t p s : / / d o i . o r g/10.1186/1471-2458-13-353

Haley, C. A., Cain, K. P., Yu, C., Garman, K. F., Wells, C. D., \& Laserson, K. F. (2008). Risk-based screening for latent tuberculosis infection. Southern Medical Journal, 101(2), 142-149. https://doi.or-

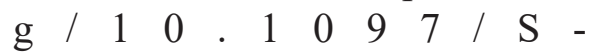
MJ.0b013e3181611c9f

Hauck, F. R., Neese, B. H., Panchal, A. S., \& El-Amin, W. (2009). Identification and management of latent tuberculosis infection. American Family Physician, 79(10), 879-886.

Joshi, R., Reingold, A. L., Menzies, D., \&

Pai, M. (2006). Tuberculosis among Health-Care Workers in Low- and Middle-Income Countries: A Systematic Review. PLoS Medicine, 3(12), e494. https://- 
Sunny Sánchez-Giler, Dolores Zambrano-Castro, Magister en Microbiología. Docente Universidad, Debbie Nath- Lynch , Andrea Murillo-Soriano, Juan Murillo-Zambrano , Gregorio Ortiz-Bermudez, Ma. Fernanda Sánchez-Carriel, Roberto Oleas-Narea, Genaro Cedeño-Gutiérrez, Walter Noboa-Quimí, Doménica Sotomayor-Salvatierra, Doménica Santamaría-Obando

doi.org/10.1371/jour nal.pmed.0030494

Lee, J. E., Kim, H.-J., \& Lee, S. W. (2011). The clinical utility of tuberculin skin test and interferon- $\gamma$ release assay in the diagnosis of active tuberculosis among young adults: a prospective observational study. BMC Infectious Diseases, 11(1), 1-7. https://doi.or$\mathrm{g} / 10.1186 / 1471-2334-11-96$

Legesse, M., Ameni, G., Mamo, G., Medhin, G., Bjune, G., \& Abebe, F. (2011). Community-based cross-sectional survey of latent tuberculosis infection in Afar pastoralists, Ethiopia, using QuantiFERON-TB Gold In-Tube and tuberculin skin test. BMC Infectious Diseases, 11, 89. https://doi.org/10.1186/1471-2334-11-89

Menzies, D. (2000). What does tuberculin

reactivity after bacille Calmette-Guerin vaccination tell us? Clinical Infectious Diseases: An Official Publication of the Infectious Diseases Society of America, 31 Suppl 3, S71-74. https://doi.or$\mathrm{g} / 10.1086 / 314075$

Nienhaus, A., Schablon, A., Preisser, A. M., Ringshausen, F. C., \& Diel, R. (2014). Tuberculosis in healthcare workers - a narrative review from a German perspective. Journal of Occupational Medicine and Toxicology (London, England), 9(1), 9. h t t p s : / / d o i . o r g/10.1186/1745-6673-9-9
OMS. (2014a). Tuberculosis (TB).

Recuperado de http://www.who. int/gho/tb/en/

OPS. (2014). Tuberculosis. Recuperado de http://www.pahoindex.php? option $=$ com_content $\&$ view $=$ article\&id=1162:marzo-24-2014\&Itemid $=356$

Pai, M., Dendukuri, N., Wang, L., Joshi, R., Kalantri, S., \& Rieder, H. L. (2008). Improving the estimation of tuberculosis infection prevalence using T-cell-based assay and mixture models. The International Journal of Tuberculosis and Lung Disease: The Official Journal of the International Union against Tuberculosis and Lung Disease, 12(8), 895-902.

Pai, M., Gokhale, K., Joshi, R., Dogra, S., Kalantri, S., Mendiratta, D. K., ... Colford, J. M. J. (2005). Mycobacterium tuberculosis infection in health care workers in rural India: comparison of a whole-blood interferon gamma assay with tuberculin skin testing. JAMA, 293(22), 2746-2755. https://doi.org/10.1001/jama.293.22.2746

Pai, M., Zwerling, A., \& Menzies, D.

(2008). Systematic review: T-ce11-based assays for the diag- nosis of latent tuberculosis infec- tion: an update. Ann Intern Med, 149. http://doi.org/10.7326/003-48 19-149-3-200808050-00241

Rafiza, S., Rampal, K. G., \& Tahir, A. 
(2011). Prevalence and risk factors of latent tuberculosis infection among health care workers in Malaysia. BMC Infectious Diseases, 11(1), 1-7. https://doi.org/10.1186/1471-2334-11-19

Regatieri, A., Abdelwahed, Y., Perez, M., \& Bush, L. (2011). Testing for Tuberculosis: The Roles of Tuberculin Skin Tests and Interferon Gamma Release Assays. Recuperado a partir de http://www.medscape.com/viewarticle/734337_2

Ringshausen, F. C., Schablon, A., \& Nienhaus, A. (2012). Interferongamma release assays for the tuberculosis serial testing of health care workers: a systematic review. Journal of Occupational Medicine and Toxicology (London, England), 7(1), 6. https://doi.or$\mathrm{g} / 10.1186 / 1745-6673-7-6$

Schablon, A., Beckmann, G., Harling, M., Diel, R., \& Nienhaus, A. (2009). Prevalence of latent tuberculosis infection among health care workers in a hospital for pulmonary diseases. Journal of Occupational Medicine and Toxicology (London, England), 4, 1.ht$\mathrm{t} \mathrm{p}:$ : / $/ \mathrm{d}$ o $\mathrm{i}$. $\mathrm{o} \quad \mathrm{r}$ g/10.1186/1745-6673-4-1

Shakak, A. O., Khalil, E. A. G., Musa, A. M., Salih, K. A. E. M., Bashir, A. E. A., Ahmed, A. H., \& Elhassan, A. M. (2013). Prevalence of latent tuberculosis infection in Sudan: a case-control study comparing interferon-gamma release assay and tuberculin skin test. $B M C$ Public Health, 13, 1128. https://d o i . o r r $\mathrm{g} / 10.1186 / 1471-2458-13-1128$

Sharma, S. K., Mohanan, S., \& Sharma, A. (2012). Relevance of latent TB infection in areas of high TB prevalence. Chest, 142(3), 761-773. https://doi.org/10.1378/chest.12-0142

van Zyl Smit, R. N., Pai, M., Yew, W. W., Leung, C. C., Zumla, A., Bateman, E. D., \& Dheda, K. (2010). Global lung health: the colliding epidemics of tuberculosis, tobacco smoking, HIV and COPD. The European Respiratory Journal, 35(1), 27-33. https://doi.org/10.1183/09031936.00072909

Whitaker, J. A., Mirtskhulava, V., Kipiani, M., Harris, D. A., Tabagari, N., Kempker, R. R., \& Blumberg, H. M. (2013). Prevalence and incidence of latent tuberculosis infection in georgian healthcare workers. PloS One, 8(3), e58202. https://doi.org/10.1371/journal.pone.0058202

Winje, B. A., Oftung, F., Korsvold, G. E., Mannsaker, T., Jeppesen, A. S., Harstad, I., ... Heldal, E. (2008). Screening for tuberculosis infection among newly arrived asylum seekers: comparison of QuantiFERONTB Gold with tuberculin skin test. BMC Infectious Diseases, 8, $65 . \quad$ https://doi.or- 


\section{$\mathrm{g} / 10.1186 / 1471-2334-8-65$}

World Health Organization. (2014b).

World Health Organization website. Recuperado a partir de http://ap p s.who.int/iris / b i t s tream/10665/112738/1/978924069 2671_eng.pdf?ua $=1$

Yunga, J. (2014). Anuario de Estadística de Salud: Recurso y Actividades 2014. Recuperado a partir de http://www.ecuadorencifras.gob.ec/documentos/web-inec/Es$\begin{array}{lllll}\mathrm{a} & \mathrm{d} & \mathrm{i} & \mathrm{s} & -\end{array}$ ticas_Sociales/Recursos_Activida des_de_Salud/Publicaciones/Anua rio_Rec_Act_Salud_2014.pdf 